\title{
Nano-structured natural bentonite clay coated by polyvinyl alcohol polymer for gamma rays attenuation
}

\author{
Ibrahim Z. Hager ${ }^{1} \cdot$ Yasser S. Rammah ${ }^{1} \cdot$ Hossam A. Othman ${ }^{1} \cdot$ Eman M. Ibrahim ${ }^{2}$ (D) Sayed F. Hassan ${ }^{3}$. \\ Fawzy H. Sallam ${ }^{2}$
}

Received: 29 October 2018 / Accepted: 5 May 2019 / Published online: 11 May 2019

(c) The Author(s) 2019

\begin{abstract}
The main goal of this work is to find natural rock materials that can be used as effective gamma rays shielding at minimal cost, reliability and wide applications. It must be at particular weight and volume (lighter and more protection). Natural bentonite clay can be used as shelters from nuclear waste because of its large availability and low cost. Bentonite clay was used in two forms naturally as it is from its ores and in ground phase. Natural bentonite was cut into cylindrical pellets at different thicknesses; also, the other form pressed into cylindrical pellets with different thicknesses and different pressing pressures (50, 100 and $150 \mathrm{bar})$. The different samples are coated with polyvinyl alcohol polymer to prevent nuclear waste leakage through porosity of clay. Chemical analysis and density are measured for all samples. Bentonite clay was found naturally in nanometer scale because it is formed from volcanic ash deposits. The nanoparticle size was determined by dynamic light scattering and Williamson-Hall size analysis using XRD patterns and the help of X-powder program. The particle size of natural bentonite was found to be $59.79 \mathrm{~nm}$. The microstructure was characterized by scanning electron microscope and transmission electron microscopy. The linear and mass attenuation coefficients of nano-structured bentonite clay (natural and pressed) were determined at $662 \mathrm{keV}$ energy of ${ }^{137} \mathrm{Cs}$; at 1173 and $1332 \mathrm{keV}$ energies of ${ }^{60} \mathrm{Co}$, gamma ray sources were determined by using $\mathrm{NaI}(\mathrm{Tl})$ scintillation detector. The experimental results showed that the ground bentonite pressed at 150 bar gave the highest linear and mass attenuation coefficients than other samples. The theoretical and the experimental calculations of mass attenuation coefficient were found to be in a good agreement.
\end{abstract}

Keywords Bentonite clay $\cdot$ Polyvinyl alcohol $\cdot$ Nanosize bentonite $\cdot$ Gamma rays attenuation

\section{Introduction}

The rapid development in science technology makes nuclear technology widely used in electricity generation, industry and medical care, which have increased people's contact with different kinds of radiation [1-4]. Three main methods for protection from radiation are usually utilized; these are time, distance and shielding. Among the three methods, shielding is the most important in which shielding materials

Eman M. Ibrahim

yousif_eman@yahoo.com

1 Department of Physics, Faculty of Science, Menoufia University, Shibin El Kom, Egypt

2 Department of Geochemical Exploration, Nuclear Materials Authority, Cairo, Egypt

3 Department of Medical and Radiological Research, Nuclear Materials Authority, Cairo, Egypt become important [5]. Different radiation protection materials were developed in order to reduce the harm of radiation to the human body. Concrete and lead products have been widely used in fixed-type nuclear reactors and accelerator protection; however, nuclear waste transport, storage containers and space vehicles require a particular weight and volume, so nanosize shielding material reduces thickness used. Natural materials can be used as a shelters from nuclear waste because of its availability and low cost [1-4].

The lighter and more efficient materials of radiation protection have been required occasions. Due to the low density and the easy processing characteristics, a new type of material which is a polymer-based compound material filled with radiopaque powder is now becoming more and more popular. It has good thermodynamic and structural properties, especially its good radiopaque ability materials; also, it prevents nuclear waste leakage from clay porosity $[6,7]$. In performance of radiation shielding, polymer materials are 
inferior to metals, but there are merits in flexibility, workability, chemical stability, low cost, volume reduction after use, etc. [8]. Polyvinyl alcohol (PVA) is essentially made from polyvinyl acetate through hydrolysis. PVA is an artificial polymer that has been used during the first half of the twentieth century worldwide. It has been applied in the industrial, commercial, medical and food sectors and has been used to produce many end products, such as lacquers, resins, surgical threads and food packaging materials that are often in contact with food [9]. PVA polymer is selected due to its amazing properties such as high optical transmission, water solubility, stable thermal, nontoxic and noncorrosive nature that makes it a good matrix for optoelectronic and a variety of other applications [10-12].

Today's application of radiation sources and radioactive materials in various fields, such as nuclear power plants, nuclear medicine, as well as industry and agriculture, has made it essential to study different parameters related to shielding against harmful and dangerous radiations [13]. Various materials which are used for shielding include lead, copper, bismuth, steel, concretes and organic compounds such as oils, paraffins, plastics and rubber. The shields could take different forms like blocks, plates, rods, pellets, etc., which can act as fillers for ducts, trenches and penetrations. Shielding pellets are useful in areas that are irregular in shapes or inaccessible to personnel. These can be poured into places or transported by air pressure or vacuum [14].

A number of experimental and theoretical works have been performed on radiation shielding, which has large different application areas with different materials such as bricks [15], ores [16, 17], glasses [18, 19], organic compounds [20], minerals [21], soil [22, 23]), rocks [24-27], concretes [28, 29] and building materials [30, 31]. Variety of rocks and concretes are used in the radiation shielding technology because of its high attenuation cross section for $\mathrm{X}$-rays, gamma ray photons and neutrons. For shield designs, gamma ray was one of the main types of nuclear radiation, which have to be considered. The type and amount of shielding required depend on the type of radiation, the activity of the radiation source and the dose rate that is acceptable for outside the shielding material. However, there are other factors for choice of shielding material such as their cost and weight [32]. Therefore, in this study, natural bentonite rocks have been used for gamma ray shielding.

The objective of this study is to seek more efficient gamma rays shielding material reliable in radiation research. For this purpose, the linear and mass attenuation coefficients of $\gamma$ - rays for four samples (natural bentonite, pressed bentonite at 50, 100 and 150 bar) were measured at energies of 662,1173 and $1332 \mathrm{keV}$. The calculated values were used to estimate another parameter such as half-value layer, tenth value layer and mean free path. For the comparison, mass attenuation coefficient of lead, which is commonly used for radiation shielding, has been calculated in the same setup to investigate the shielding ability of natural and pressed bentonite to lessen gamma radiation.

\section{Theoretical aspect}

The linear attenuation coefficient can be calculated according to The Lambert-Beer law which describes attenuation of a monoenergetic beam as follows:

$I=I_{\mathrm{o}} e^{-\mu x}$

where $I=$ transmitted gamma radiation intensity, $I_{\mathrm{o}}=$ incident gamma radiation intensity, $x=$ is the thickness of the absorbing medium, $\mu=$ is the linear attenuation coefficient. For photons in an attenuating medium, the mass attenuation coefficient $\left(\mu_{\mathrm{m}}\right)$ is given by:

$\mu_{\mathrm{m}}=\ln \left(\frac{I_{\mathrm{o}}}{I}\right) / \rho x$

where $\rho$ is the density of the shield material [33]. The mass attenuation coefficient $(\mu / \rho)$ is a measure of probability of interaction that occurs between incident photons and matter per unit mass per unit area [34].

The effectiveness of gamma rays shielding is described in terms of the half-value layer (HVL) and the tenth value layer (TVL) of a target attenuator material. HVL is defined as the thickness of the absorber material that reduces the intensity of gamma radiation to half of its value. It depends on absorber material and photon energy of the radiation. Also, tenth value layer (TVL) is defined as the thickness of the absorber material that reduces gamma rays to one-tenth of its original intensity [35].

$\mathrm{HVL}=\mathrm{Ln} 2 / \mu$

$\mathrm{TVL}=\mathrm{Ln} 10 / \mu$

The last shielding factor is mean value path which defined as the distance between two successive interactions; mathematically, it is the inverse of the linear attenuation coefficient. The relation between it and photon energy defines why the numbers of interactions become higher when distance between the interactions gets smaller. The mean free path (mfp) was calculated for shielding material using the following equation [13]:

$\operatorname{mfp}=\frac{1}{\mu}$

\section{Materials and methods}

Natural bentonite is brought from El-Mutalla Mountain located in southwestern Sinai, Egypt. This natural bentonite sample was cut into cylindrical pellets with different thicknesses to produce the first form. Natural bentonite is 
manually crushed by hummer and ground by ball milling (Fritsch pulverisette, model 02.102) for $1 \mathrm{~h}$. It was pressed into cylindrical pellets with different thicknesses and different pressing pressures 50,100 and 150 bar producing the second form of bentonite. The two forms differ from each other in pressing pressure so we can know on what pressure natural bentonite formed environmentally by comparing their attenuation coefficients. Different thicknesses of the two forms of bentonite clay are shown in Fig. 1 for natural and pressed samples. The two forms of bentonite clay are coated by polyvinyl alcohol polymers (PVA) which produce matrix samples. The weight of the deposited PVA on different bentonite clay pellets represents a $10 \%$ from the whole weight for each matrix sample, while the weight of each bentonite clay pellets represents a $90 \%$ from this matrix. Therefore, the samples after coating by PVA are ready for measuring attenuation coefficients.

The attenuation coefficients of each sample were measured for gamma rays of $662 \mathrm{keV}$ energy of ${ }^{137} \mathrm{Cs} ; 1173$ and $1332 \mathrm{keV}$ energies of ${ }^{60} \mathrm{Co}$ using a well-calibrated gamma ray spectrometer which consists of $3^{\prime \prime} \times 3^{\prime \prime} \mathrm{NaI}(\mathrm{Tl})$ scintillation detector. The detector is protected by a copper cylindrical protection $(0.6 \mathrm{~cm}$ thickness) against induced $\mathrm{X}$-rays and a chamber of lead bricks against the environmental radiations, and then, the detector is covered by a lead cover ( $5 \mathrm{~cm}$ thickness). The detector is connected with Nuclear Enterprises main shaping amplifier and Tennelec high voltage-power supply with $\mathrm{HV}$ digital display. The detector is also connected with Nuclease PCA- 8000 computer-based, 8192 multichannel analyzer with color graphical display of spectra and high-level technical operation features.

To accurately account for the absorption of gamma radiation in the investigated samples, the narrow beam geometry was applied in which lead collimator is used as shown in
Fig. 2. The cylindrical pellet samples were stacked in the lead collimator which placed on the cap of the detector with the source placed above a hole aligned to the middle of the sample pellet to ensure that the detector received the collimated beam only through the sample layer. Under this geometry, the narrow beam of monoenergetic photons of intensity $\mathrm{I}_{\mathrm{o}}$ and transmitted I photon was measured without and with the sample pellet in place for time duration of $1800 \mathrm{~s}$.

\section{Results and discussion}

The natural bentonite clay was characterized by dynamic light scattering (DLS) technique, X-ray diffraction (XRD) analysis, scanning electron microscopy (SEM) and transmission electron microscopy (TEM).

The radiation must be attenuated enough to protect the personnel from the harmful effects caused by it and

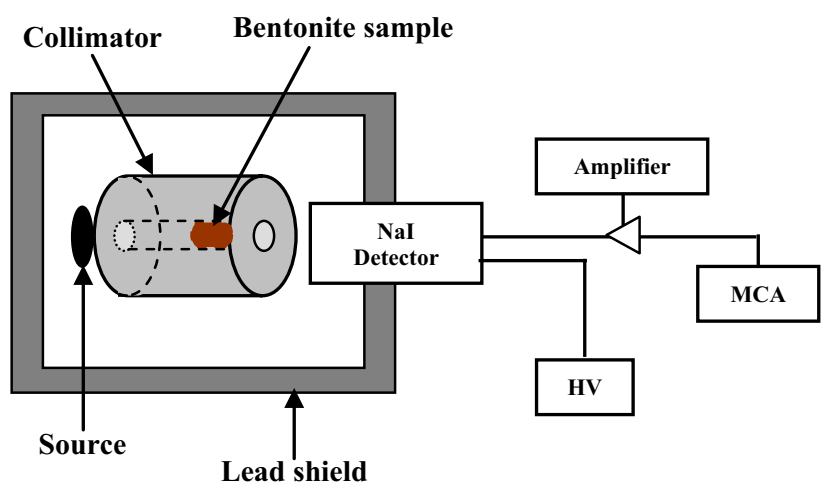

Fig. 2 The experimental setup of the narrow beam Transmission method
Fig. 1 Different thicknesses of the two forms of bentonite clay; a natural $\mathbf{b}$ ground and pressed
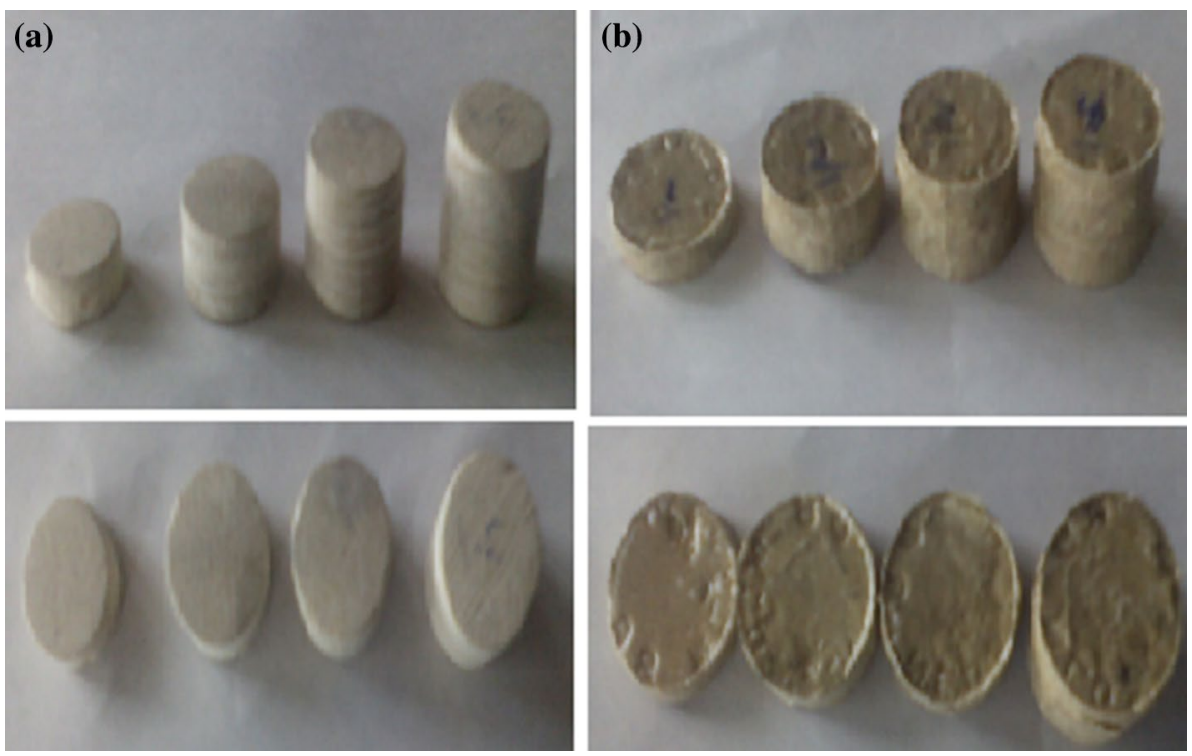
also enable them to work by using an opposite shielding material. To design and choose an appropriate shielding, it is necessary to have known its nuclear, structural and physical properties and also the characteristics of radiation impinging on it. The nuclear parameters that must be known to design and choose a shielding material are linear attenuation coefficient $(\mu)$, total mass attenuation coefficient $\left(\mu_{\mathrm{m}}\right)$, for gamma rays which is related to halfvalue layer (HVL), tenth value layer (TVL) and mean free path (mfp) [36].

Gamma ray shielding properties are calculated in terms of density, linear attenuation coefficient, mass attenuation coefficient $(\mu / \rho)$, half-value layer parameter (HVL) and mean free path (MFP) at photon energies 662, 1173 and $1332 \mathrm{keV}$. Shielding properties of the two forms of nano-structured bentonite clay (natural and pressed) have been investigated against gamma rays using three energies 662, 1173 and $1332 \mathrm{keV}$ emitted by ${ }^{137} \mathrm{Cs}$ and ${ }^{60} \mathrm{Co}$ point sources.

\section{Characterization of bentonite clay}

\section{Chemical composition analysis of bentonite clay}

The two forms of bentonite clay have the same chemical composition, but the only difference between them is pressing pressure. As observed in Table 1, the amount of water and organic matter found in bentonite clay is $30.34 \%$ of all bentonite content, as loss of ignition (L.O.I). There is a variety of clay oxide minerals in bentonite clay structure so it is considered a mixture of elements which can be used as a composite shielding material against gamma rays.

\section{Particle size measurements}

Dynamic light scattering (DLS) analysis The particle size of natural bentonite clay is determined using dynamic light scattering (DLS) technique. Figure 3 shows that natural bentonite is in nano-sized scale but has large particle size distribution, this because of agglomeration of nanoparticles when water had been used in suspension. Nanoparticles have high surface energy causing them to agglomerate until they are stable [37]. It needs surfactant that prevents agglomeration. The lowest size in Fig. 3 is $122 \mathrm{~nm}$ and represents $75 \%$ from all powder structure which lies between 122.4 and $220 \mathrm{~nm}$. As a result of the presence of agglomeration and precipitation of nanoparticles during DLS measurements, another method is needed for more specific determination.

Williamson-Hall (W-H) size analysis The crystallite size and lattice strain were estimated by Williamson-Hall (W-H) equation given by the following equation:

$\beta \cos \theta=\frac{K \lambda}{D}+4 \varepsilon \sin \theta$

where $\beta$ is full width at half-maxima (FWHM) in radians, $\theta$ is the diffraction angle, $K$ is the shape factor $(K=0.9), \lambda$ is the wavelength of $\mathrm{X}$-rays, $D$ is the crystallite size and $\varepsilon$ is strain [38].

The XRD pattern of natural bentonite clay is shown in Fig. 4. This natural bentonite clay contained two crystalline phases: calcite $\left(\mathrm{CaCO}_{3}\right.$, JCPDS card No. 00-005-0586) and quartz $\left(\mathrm{SiO}_{2}\right.$, JCPDS card No. 898935) which represent
Table 1 Chemical composition analysis for natural bentonite clay

Fig. 3 DLS analysis for natural bentonite clay

\begin{tabular}{llllllllllll}
\hline Oxides (\%) & $\mathrm{SiO}_{2}$ & $\mathrm{Al}_{2} \mathrm{O}_{3}$ & $\mathrm{P}_{2} \mathrm{O}_{5}$ & $\mathrm{TiO}_{2}$ & $\mathrm{Fe}_{2} \mathrm{O}_{3}$ & $\mathrm{CaO}$ & $\mathrm{MgO}$ & $\mathrm{Na}_{2} \mathrm{O}$ & $\mathrm{K}_{2} \mathrm{O}$ & L.O.I & Total \\
\hline Natural bentonite & 30 & 13.5 & 0.34 & 0.34 & 3.2 & 15.2 & 4.03 & 1.4 & 0.61 & 30.34 & 98.96 \\
\hline
\end{tabular}

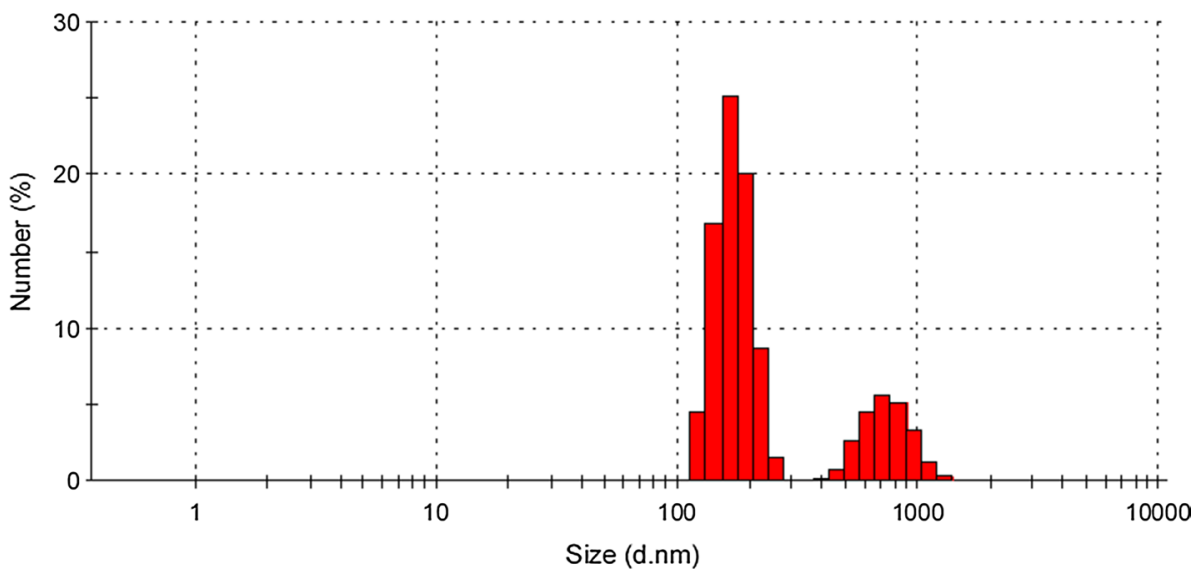


$87.6 \%$ and $12.4 \%$, respectively. The dominant (104) reflection of calcite can be found in the sample at $29.5^{\circ}$ (Fig. 4). Furthermore, the weaker (012)-, (110)-, (113)-, (202)-, (018)-, (116)-, (211)- and (122)-reflections of calcite are present at $23.1^{\circ}, 36.0^{\circ}, 39.4^{\circ}, 43.2^{\circ}, 47.2^{\circ}, 48.6^{\circ}, 56.6^{\circ}$ and $57.4^{\circ}$, respectively. It can be observed from the pattern that the most intense crystalline quartz peak at $2 \theta=26.6^{\circ}(011)$ belongs to hexagonal structure. The diffraction peaks were in good agreement with the results reported in the literature [39-43].

XRD analysis can be utilized to evaluate particle size and lattice strain due to dislocation or size reduction in the bentonite clay. Williamson-Hall analysis is used for this purpose. Figure 5 shows a Williamson-Hall plot of natural bentonite clay. The graph is plotted with the help of X-powder software and uses the Gaussian linear fitting method. The advantage of $\mathrm{W}-\mathrm{H}$ analysis is that it determines particle size and lattice strain found in the bentonite clay powder as a result of size reduction [44]. The nonuniform lattice strain of bentonite clay powder is equal to 0.168 .

Nanoscience is the study of the basic principles of molecules and structures with dimensions between 1 and $100 \mathrm{~nm}$, and nanotechnology is the most modern technological world and by having special characteristics is proposed to be used in all fields of science and technology [45]. The result of $\mathrm{W}-\mathrm{H}$ analysis for the natural bentonite clay gives value of particle size as $59.79 \mathrm{~nm}$.

The natural bentonite is in nanosize scale because it is formed from deposits of volcanic ashes as a result of volcanic eruptions. Volcanic ashes consider non-intentionally made nanomaterials which refer to nano-sized materials found naturally in the environment [46].

Nanomaterials due to their small size can be physical or chemical properties than the same materials in the micron scale. The most important feature of these materials is that

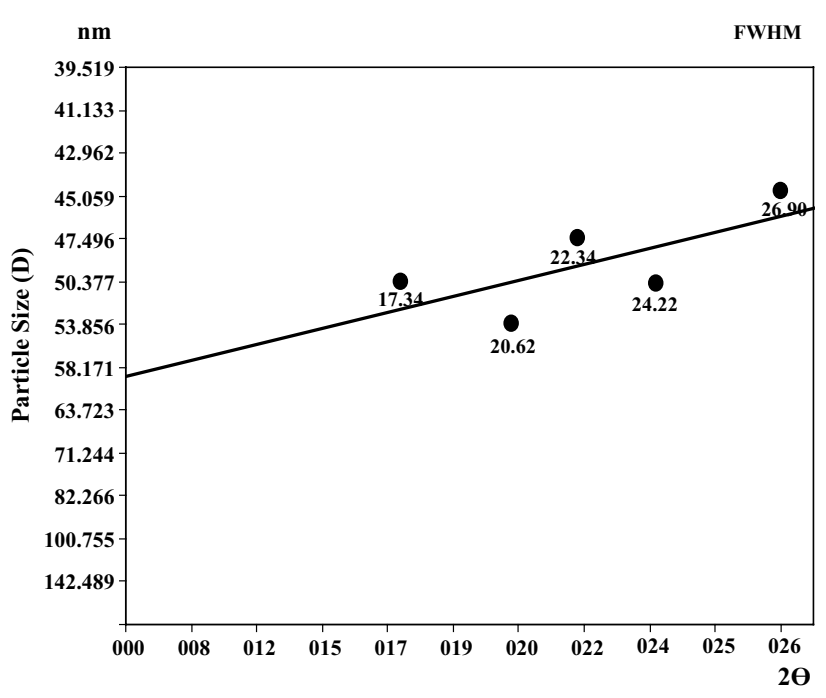

Fig. 5 Particle size of natural bentonite using W-H analysis

they are caused by different properties, a high surface to volume ratio. According to studies, it appears that nanoscale particles can cause greater protection against ionizing radiation [45].

\section{Scanning and transmission electron microscope}

Numerous microscopy techniques are commercially available; however, scanning electron microscopy (SEM) and transmission electron microscopy (TEM) are arguably the most popular for nanoparticle analysis. The morphological properties of the natural bentonite clay surface were investigated using scanning electron microscope (SEM) and transmission electron microscope (TEM). SEM explains that particle size is nearly uniform and spherical throughout the
Fig. 4 XRD pattern of natural bentonite clay

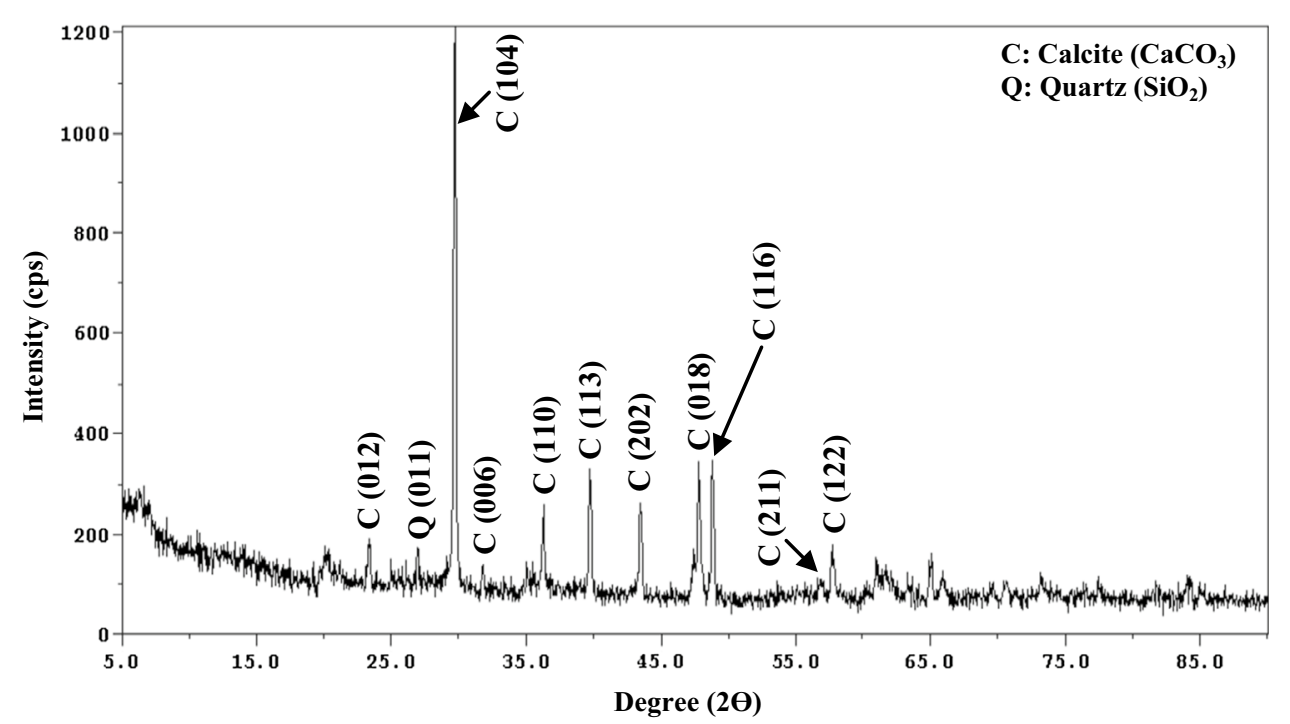


surface, and fine grain size of the natural bentonite clay was observed (Fig. 6a). TEM showed that the particle size of the natural bentonite clay was in nanometer scale with distribution between $69.14 \mathrm{~nm}$ to $101.46 \mathrm{~nm}$ (Fig. 6b). However, the particle size calculated from $\mathrm{W}-\mathrm{H}$ method is $59.79 \mathrm{~nm}$, and this variation in size from $\mathrm{W}-\mathrm{H}$ to TEM could be caused by the presence of strain. Natural nanoparticles like nanobentonite clay produce more uniform distribution inside the matrix relative to micro particles and consequently higher photon attenuation.

\section{Density measurements}

The physical densities of the corresponding samples were measured by the conventional method [23]. The density of all bentonite clay samples [natural, pressed at 50,100 and $150 \mathrm{bar}$ ] is calculated and plotted as shown in Fig. 7. The measured density of the natural clay is equal to $0.85 \mathrm{~g} / \mathrm{cm}^{3}$. The density increases from 0.85 to $1.122 \mathrm{~g} / \mathrm{cm}^{3}$ for ground and pressed bentonite at 150 bar.

\section{Linear attenuation coefficient}

The measured gamma photons emitted from ${ }^{137} \mathrm{Cs}$ to ${ }^{60} \mathrm{Co}$ point sources without and with the bentonite absorber material [natural, pressed $(50,100$ and 150 bar)] at different thicknesses and different energies are illustrated in Table 2. The total linear attenuation coefficient $(\mu)$ of the absorber for $\gamma$-rays of appropriate energy can be evaluated from a linear graph of $\ln \left(I_{\mathrm{o}} / I\right)$ versus thickness " $x$ " of the absorber (Fig. 8) where the slope of the line represents the attenuation coefficient. It is clear that the attenuation of gamma rays increased with increasing the thickness of bentonite clay for different energies. The linear attenuation coefficients are calculated from the graphs for the two forms of bentonite clay samples (natural and pressed) at different energies and are compared with other worldwide (Table 3).

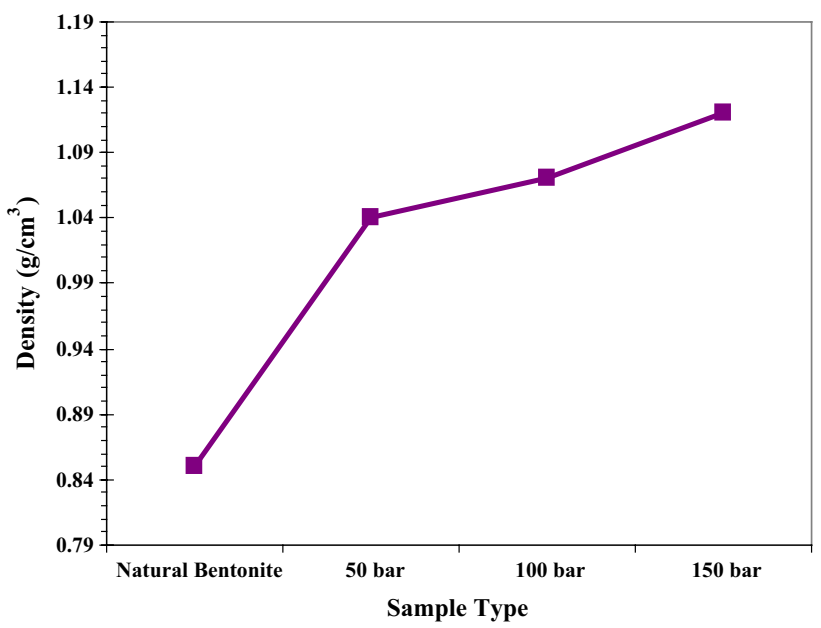

Fig. 7 Density of ground bentonite clay versus pressure of pressing

It is clearly seen in Fig. 9 that the pressed bentonite samples at 150 bar have the high attenuation coefficients $(0.096$, 0.078 and $0.073 \mathrm{~cm}^{-1}$ ) for all photon energies compared with natural bentonite and other pressed bentonite samples at (50 and 100 bar) because they have the highest density $\left(1.12 \mathrm{~g} / \mathrm{cm}^{3}\right)$ in the studied bentonite samples. The linear attenuation of the bentonite clay forms depend on the energy of the photons that interact with it; this is due to the different photon absorption mechanisms which are photoelectric at low energy; Compton scattering at low and mid-energy range also pair production at high energy (above $1022 \mathrm{keV}$ ). The highest linear attenuation coefficient in ground (150 bar) also has the highest density.

The density and linear attenuation coefficient increase with increasing pressing pressure of bentonite clay (Figs. 7, 9), while linear attenuation coefficient decreases with increasing the energy of gamma rays (Fig. 10). Plot shown in Fig. 11 verifies the dependence of linear attenuation coefficient on density of different forms of bentonite clay.
Fig. 6 Micrograph of a SEM and $\mathbf{b}$ TEM for natural bentonite clay
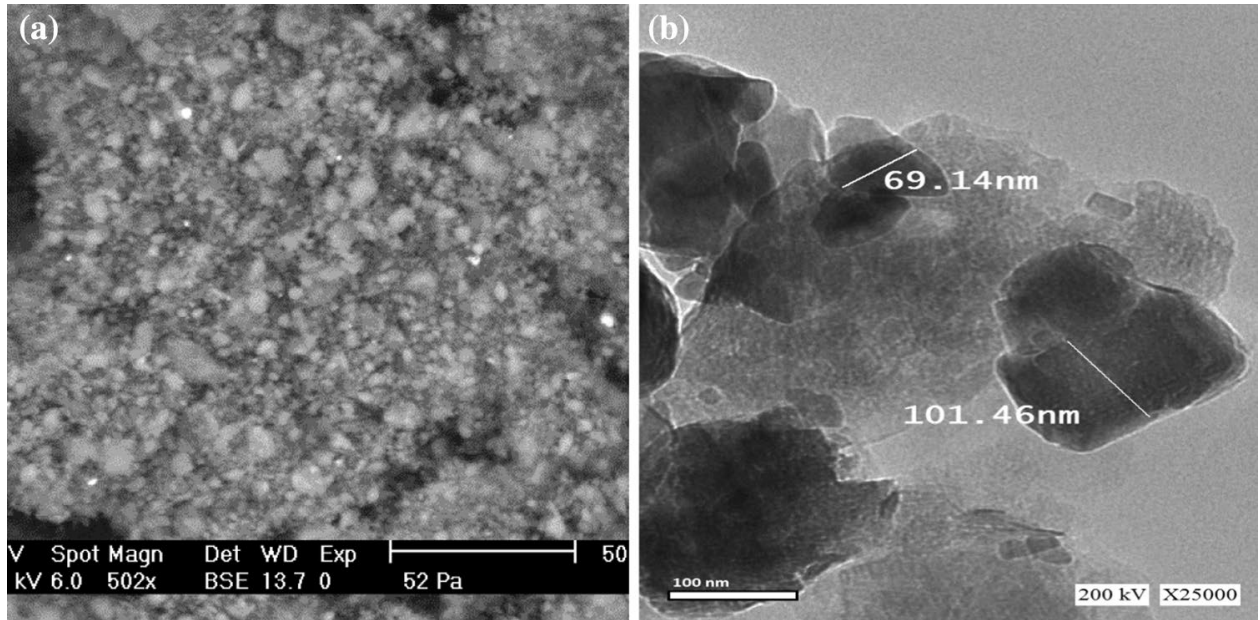
Table 2 Measured gamma photons without and with two forms of bentonite absorber material [natural, pressed (50,100 and 150 bar)] at different thicknesses and different energies

\begin{tabular}{|c|c|c|c|}
\hline \multirow[t]{2}{*}{ Thickness (cm) } & \multicolumn{3}{|c|}{ Energy (keV) } \\
\hline & 662 & 1173 & 1332 \\
\hline \multicolumn{4}{|l|}{ Natural bentonite } \\
\hline 0 & 18,189 & 2918 & 2276 \\
\hline 0.629 & 17,219 & 2784 & 2231 \\
\hline 1.079 & 16,643 & 2692 & 2153 \\
\hline 1.661 & 16,024 & 2605 & 2094 \\
\hline 2.012 & 15,619 & 2563 & 2058 \\
\hline 2.535 & 15,118 & 2523 & 2036 \\
\hline 3.000 & 14,683 & 2444 & 1974 \\
\hline \multicolumn{4}{|c|}{ Pressed bentonite (50 bar) } \\
\hline 0 & 18,412 & 2882 & 2070 \\
\hline 0.498 & 17,321 & 2809 & 2065 \\
\hline 0.952 & 16,600 & 2742 & 2028 \\
\hline 1.380 & 16,116 & 2690 & 1979 \\
\hline 1.736 & 15,651 & 2610 & 1937 \\
\hline 2.076 & 15,104 & 2535 & 1870 \\
\hline \multicolumn{4}{|c|}{ Pressed bentonite (100 bar) } \\
\hline 0 & 17,759 & 3161 & 2134 \\
\hline 0.506 & 16,921 & 3045 & 2082 \\
\hline 0.945 & 16,447 & 2955 & 2032 \\
\hline 1.266 & 15,877 & 2887 & 2007 \\
\hline 1.764 & 15,050 & 2797 & 1934 \\
\hline 2.069 & 14,883 & 2740 & 1875 \\
\hline \multicolumn{4}{|c|}{ Pressed bentonite (150 bar) } \\
\hline 0 & 18,362 & 3062 & 1628 \\
\hline 0.450 & 17,414 & 2951 & 1588 \\
\hline 0.874 & 16,836 & 2873 & 1550 \\
\hline 1.365 & 16,274 & 2772 & 1501 \\
\hline 1.660 & 15,648 & 2690 & 1472 \\
\hline 1.902 & 15,054 & 2638 & 1419 \\
\hline
\end{tabular}

Moreover, polyvinyl alcohol polymer (PVA) protects the studied bentonite rock from the corrosion occurred by chemical weathering. PVA's resistance against organic solvents and aqueous solubility makes it adaptable for many applications. PVA is commonly used in the textile industries, for paper products manufacturing, in the food packaging industry, and as medical devices. PVA is used as an industrial and commercial product due to its low environmental impact, which includes its high chemical resistance, aqueous solubility and biodegradability. In medical devices, PVA is used as a biomaterial due to its biocompatible, nontoxic, noncarcinogenic, swelling properties and bioadhesive characteristics [48].

Moreover, some studies have been carried out on the effect of shielding materials grain size on linear attenuation coefficients. It is widely believed that nano-sized particles are able to disperse more uniformly within the matrix with fewer agglomerations when compared with micro-sized particles, thus improving the radiation attenuation ability of the materials [49]. As mentioned before, the particle size of the natural bentonite clay was measured to be $59.79 \mathrm{~nm}$. Therefore, this particle size plays an important role in the attenuation of radiation. Nano-material has properties that are different from those of bulk materials. The properties of nano-material are very much different from those at a larger scale. Two principle factors cause the properties of nano-material to differ significantly from other material; (1) increased relative surface area and (2) quantum confinement effect [50]. The effectiveness of nanoparticles is that decreasing pores inside material structure that produces regular distribution and increase the electron density of the material sample; this leads to increase in the probability of interaction between gamma rays and shield material which increase attenuation coefficient.

\section{Mass attenuation coefficient}

Figure 12 clearly explains the variation of mass attenuation coefficients of all bentonite forms with incident photon energies. $\mu_{\mathrm{m}}$ coefficients attain their maximum values at lower energies, where photoelectric effect dominates, and decrease sharply with increasing energy. This indicates that the cross sections of gamma interactions with the electrons of the sample material increased at low energies. As the photon energy increases above $100 \mathrm{keV}, \mu_{\mathrm{m}}$ coefficients change in a narrow range for all samples and show a less energy-dependent behavior. This is a direct consequence of the Compton effect predominance at these intermediate energies. When the photon energy exceeds $1022 \mathrm{keV}, \mu_{\mathrm{m}}$ coefficients become almost constant as a result of the predominance of pair production process and the attenuation behavior becomes similar for all samples [5].

The mass attenuation coefficient shows increase in the order Natural $<$ Pressed $(50$ bar $)<$ Pressed (100 bar) < Pressed (150 bar). As pressing pressure increases, density increases and linear attenuation increases also mass attenuation increases.

Mass attenuation coefficient of the bentonite pressed at 150 bar was $0.0855 \mathrm{~cm}^{2} / \mathrm{g}$ (highest value of the calculated $\left.\mu_{\mathrm{m}}\right)$ at energy $662 \mathrm{keV}$. It is a very good shielding material. The theoretical value of mass attenuation coefficient of lead using XCOM code was $0.1101 \mathrm{~cm}^{2} / \mathrm{g}$. by comparing the value of mass attenuation coefficient of bentonite pressed at 150 bar and lead, it represents $77 \%$ from lead value at photon energy $662 \mathrm{keV}$. The value of $\mu_{\mathrm{m}}$ raised from $0.0788 \mathrm{~cm}^{2} / \mathrm{g}$ for natural bentonite to $0.0855 \mathrm{~cm}^{2} / \mathrm{g}$ for bentonite pressed at (150 bar). This value increases from 72 to $77 \%$ relative to lead at energy $662 \mathrm{keV}$. The result of this raising proves 

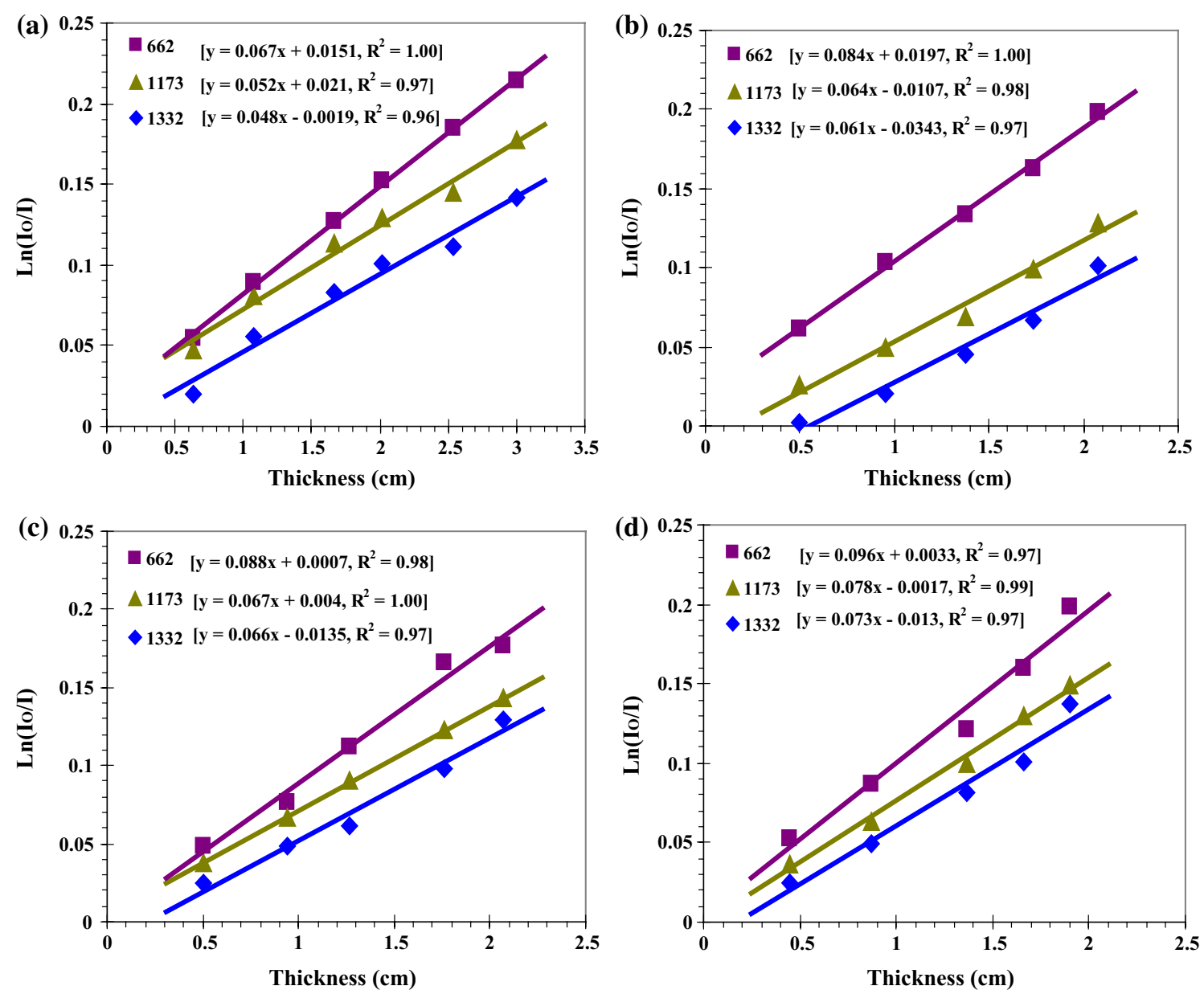

Fig. 8 Gamma transmission through a natural bentonite, pressed bentonite at b 50 bar, c 100 bar and d 150 bar at different thicknesses and different energies

the efficiency of compressing on natural bentonite clay for gamma rays attenuation.

An alternative or convenient method to experimental determination of mass attenuations coefficients is theoretical or manual calculations using tabulated data that is generated using a computer program. For this purpose, a computer program (called XCOM) was developed by Berger and Hubbell [51] for calculating cross sections and attenuation coefficients for any element, compound or mixture, at energies from $1 \mathrm{keV}$ to $100 \mathrm{GeV}$ [52]. The mass attenuation coefficients $\left(\mathrm{cm}^{2} / \mathrm{g}\right)$ of natural bentonite were theoretically calculated with the help of the XCOM program. The utilization of XCOM computer code [53], we can compare between theoretical mass attenuation coefficient and experimental one. The deviation between experimental and theoretical mass attenuation can be calculated from the relation:

Deviation $(\%)=\frac{(\text { mass attenuation })_{\text {theo }}-(\text { mass attenuation })_{\exp }}{(\text { mass attenuation })_{\text {theo }}} \times 100$
As illustrated in Table 4, the deviation values range from 0.18 to $0.63 \%$ for XCOM results. The experimental and theoretical mass attenuation coefficients were found to be in a good agreement. The discrepancies are considered not to be very large because the differences are in the range of the reported experimental errors, which is $<3.5 \%$ [13]. A comparison of the measured mass attenuation coefficient values for different kinds of samples at the same energy measured in different countries is shown in Table 5. Comparing the attenuation coefficients for different types of samples at photon energies of 1173 and $1332 \mathrm{keV}$ indicates some difference from the values measured in our study; however, at $662 \mathrm{keV}$, the difference is much smaller and is in broad agreement with the data of Gökçe et al. [47] in Turkey, Alam et al. [23] in Bangladesh and Obaid et al. [27] in India.

\section{Effective shield thickness}

Half-value layer (HVL) is an important parameter in designing the radiation shielding materials. Therefore, 
Table 3 Linear attenuation coefficients $\left(\mathrm{cm}^{-1}\right)$ of the studied samples at the investigated gamma energies in comparison with other worldwide

\begin{tabular}{|c|c|c|c|c|c|}
\hline \multirow[t]{2}{*}{ Sample type } & \multirow[t]{2}{*}{ Density $\left(\mathrm{g} / \mathrm{cm}^{3}\right)$} & \multicolumn{3}{|l|}{ Energy (keV) } & \multirow[t]{2}{*}{ References } \\
\hline & & 662 & 1173 & 1332 & \\
\hline Natural bentonite & 0.85 & 0.067 & 0.052 & 0.048 & Present work \\
\hline Pressed bentonite ( 50 bar) & 1.04 & 0.084 & 0.064 & 0.061 & \\
\hline Pressed bentonite (100 bar) & 1.07 & 0.088 & 0.067 & 0.066 & \\
\hline Pressed bentonite (150 bar) & 1.12 & 0.096 & 0.078 & 0.073 & \\
\hline \multicolumn{6}{|l|}{ Worldwide } \\
\hline High consistency concrete & Range (2.735-3.333) & $\begin{array}{l}\text { Range (0.218- } \\
0.264)\end{array}$ & $\begin{array}{l}\text { Range (0.156- } \\
0.191)\end{array}$ & $\begin{array}{l}\text { Range }(0.145- \\
0.181)\end{array}$ & Gökçe et al. [47] \\
\hline Aluminum & 2.7 & 0.224 & - & - & Reda [32] \\
\hline Iron & 7.87 & 0.585 & & & \\
\hline Copper & 8.96 & 0.638 & & & \\
\hline Lead & 11.35 & 1.211 & & & \\
\hline Beige marble (KSA) & 2.645 & 0.200 & 0.152 & 0.146 & Al-Hamarneh [5] \\
\hline Tea rose marble (Italy) & 2.616 & 0.182 & 0.146 & 0.144 & \\
\hline Rosa marble (Portugal) & 2.653 & 0.200 & 0.151 & 0.146 & \\
\hline Green marble (India) & 2.639 & 0.203 & 0.153 & 0.145 & \\
\hline Emperador marble (Spain) & 2.634 & 0.202 & 0.152 & 0.145 & \\
\hline Beige marble (Oman) & 2.608 & 0.200 & 0.149 & 0.143 & \\
\hline Polyboron & 0.971 & 0.084 & 0.064 & 0.060 & Biswas et al. [36] \\
\hline Ordinary concrete & 0.92 & 0.182 & 0.138 & 0.129 & \\
\hline Pure polyethylene & 1.19 & 0.081 & 0.062 & 0.058 & \\
\hline Borated polyethylene & 2.35 & 0.098 & 0.074 & 0.070 & \\
\hline Water & 1.0 & 0.086 & 0.066 & 0.061 & \\
\hline
\end{tabular}

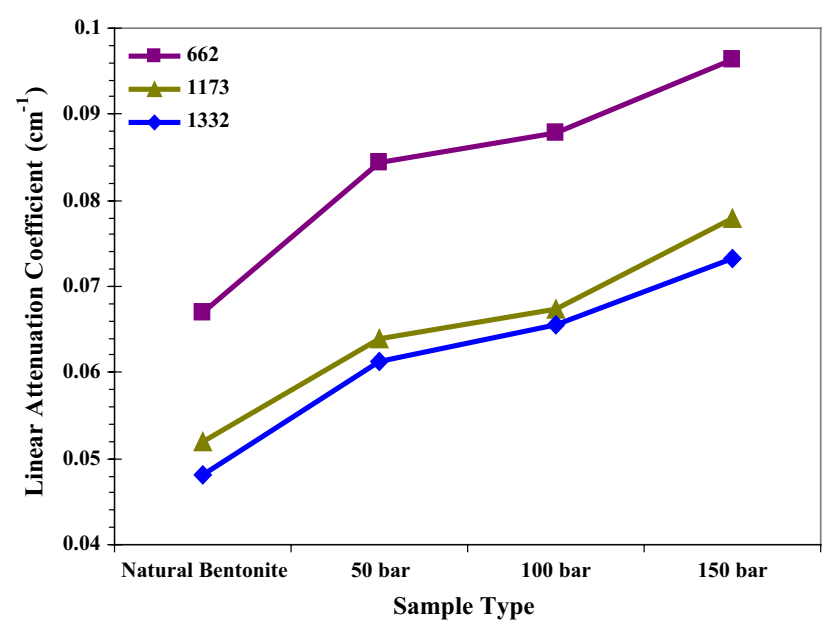

Fig. 9 Linear attenuation coefficients $\left(\mathrm{cm}^{-1}\right)$ for different energies

the shielding properties of the present materials can be investigated by studying this parameter for this type of radiation. The less the HVL of the material, the better the shielding properties it has. Thus, the shielding effectiveness of bentonite clay sample is inversely proportional to its HVL value. The energy dependence of the HVL values on photon energy is illustrated in Fig. 13. It is obvious

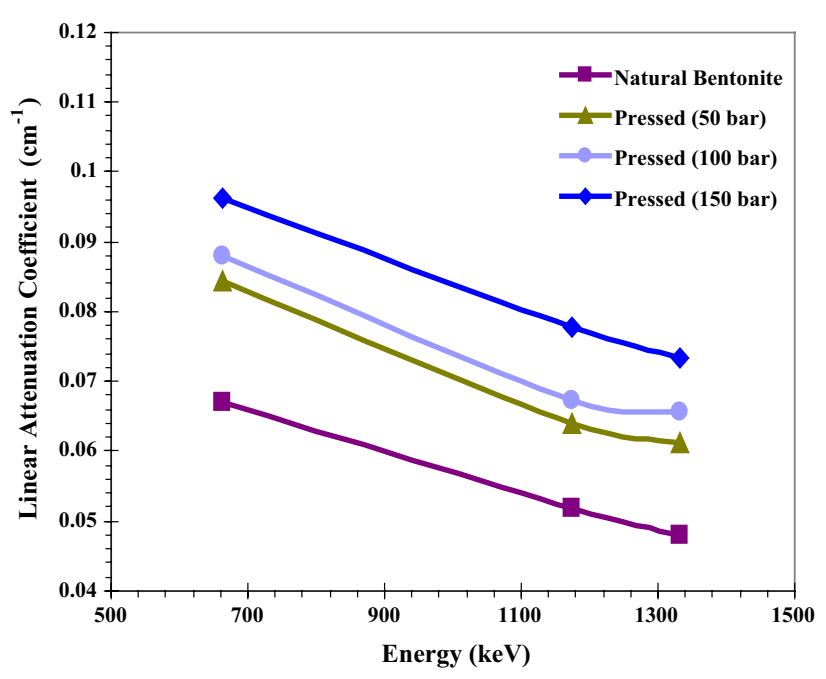

Fig. 10 Linear attenuation coefficient versus energy for different forms of bentonite clay

from the figure that the gradual increase in HVL with photon energy indicates that thicker shields are needed against high gamma radiation. The variations in HVL values may also indicate some dependence on the density of the material. In addition, Fig. 13a reveals that HVL 


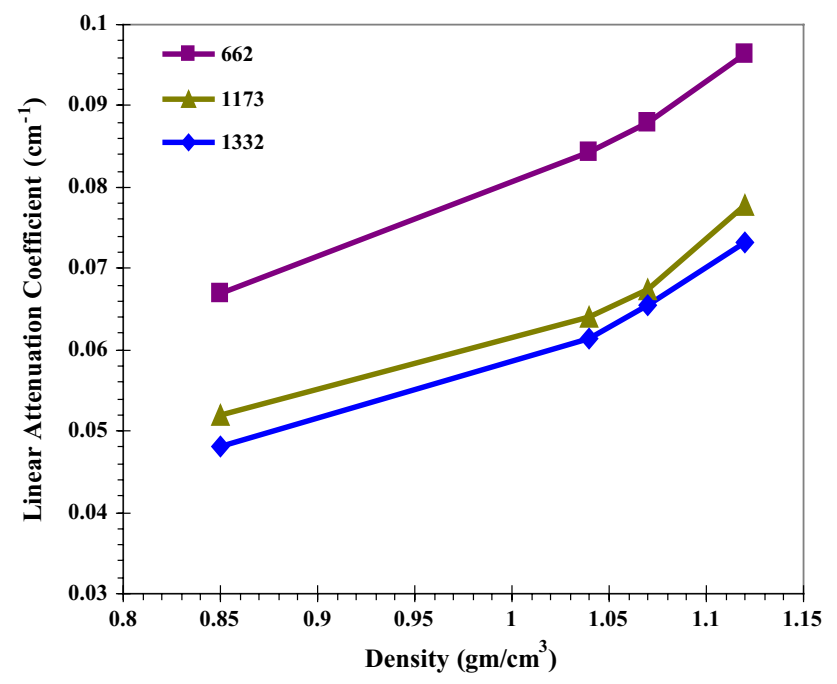

Fig. 11 Dependence of linear attenuation coefficient on density of different forms of bentonite clay

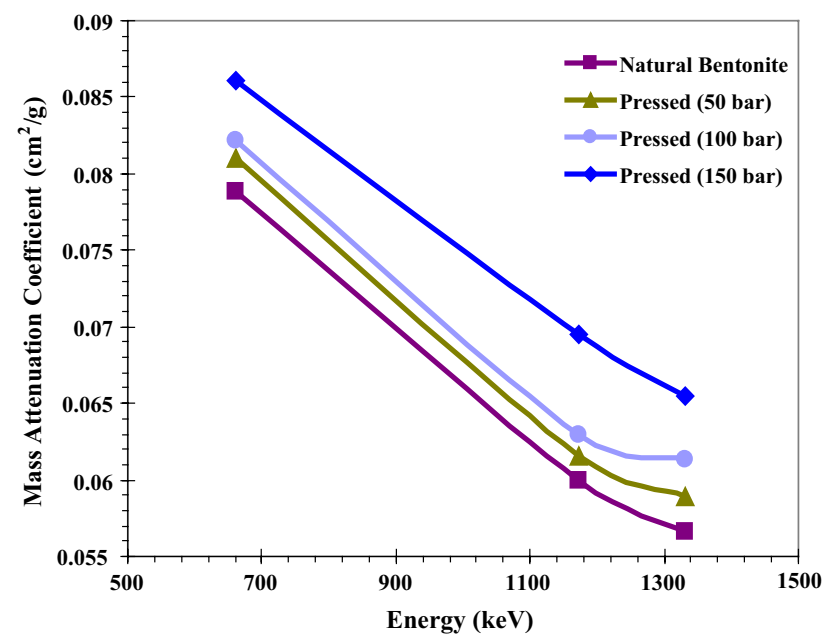

Fig. 12 Mass attenuation coefficient versus energy for different bentonite forms

Table 4 Experimental and theoretical mass attenuation coefficient $\left(\mathrm{cm}^{2} / \mathrm{g}\right)$ at different energies for natural bentonite clay and the corresponding deviation values $(\%)$

\begin{tabular}{llll}
\hline Energy $(\mathrm{keV})$ & $\mu_{\mathrm{m}}($ experimental $)$ & $\mu_{\mathrm{m}}($ theoretical $)$ & Deviation $(\%)$ \\
\hline 662 & 0.0788 & 0.0793 & 0.63 \\
1173 & 0.0600 & 0.0605 & 0.83 \\
1332 & 0.0566 & 0.0567 & 0.18 \\
\hline
\end{tabular}

parameter can be employed to distinguish between samples in terms of their shielding effectiveness. Based on that, bentonite sample pressed at 150 bar exhibited the maximum shielding effectiveness with the lowest HVL at the three incident photon energies, whereas natural bentonite sample exhibited the minimum effectiveness. As shown in Fig. 13a, b, the behavior of TVL and HVL values was found the same for different bentonite clay forms.

\section{Mean free path}

The variation of mean free path (mfp) values with energy of studied samples is shown in Fig. 14. Mean free path follows a decreasing order with increasing energy. Low values of mean free path are the indication of the increase in probability of gamma rays to get attenuated [55]. The minimum value of the mean free path is observed for ground bentonite sample pressed at 150 bar with the highest density $(1.12 \mathrm{~g} /$ $\mathrm{cm}^{3}$ ). The $\mathrm{mfp}$ values increase as gamma ray energy increase for all studied samples where low energy photon can losses its energy in a short distance while high energy one needs a large distance.

\section{Conclusion}

The toxicity of lead makes us to look for natural materials that can be used in gamma rays protection. Bentonite clay is a good choice for this purpose because of its large availability and low cost. Natural bentonite is found naturally in nanometer scale as it formed from deposits of volcanic ashes due to volcanic eruptions; this is perfect due to the effectiveness of nanometer scale materials in gamma rays shielding. The particle size of natural bentonite clay is determined as $59.79 \mathrm{~nm}$.

Natural bentonite and ground (pressed at 50, 100 and $150 \mathrm{bar}$ ) forms investigated against gamma rays, and we found that the ground bentonite is better than natural one. The developed three different ground and pressed samples (50, 100 and 150) have larger shielding factors than natural bentonite due to their higher density values than it. Ground bentonite pressed at 150 bar is the perfect choice because it has the highest linear $(\mu)$ and mass $\left(\mu_{m}\right)$ attenuation coefficients and less thickness (effective shielding) than other samples. $\mu_{\mathrm{m}}$ value of natural bentonite increases from 72 to $77 \%$ for ground (150 bar) relative to $\mu_{\mathrm{m}}$ value of lead and HVL decreases from $(10.35,13.36$ and $14.41 \mathrm{~cm})$ for natural bentonite to $(7.19,8.90$ and $9.46 \mathrm{~cm})$ for grinded $(150 \mathrm{bar})$ bentonite at photon energies $(662,1173$ and $1332 \mathrm{keV})$, respectively. Pressed bentonite sample at 150 bar can be used as shelters from nuclear waste when it coated with polyvinyl alcohol polymer to prevent leakage of nuclear waste from the porosity of bentonite clay. Moreover, polyvinyl alcohol polymer protects the studied bentonite rock from the corrosion occurred by chemical weathering. 
Table 5 Comparison of mass attenuation coefficients $\left(\mathrm{cm}^{2} / \mathrm{g}\right)$ at the studied energies for different samples with other countries

\begin{tabular}{|c|c|c|c|c|c|}
\hline \multirow[t]{2}{*}{ Sample type } & \multicolumn{3}{|l|}{ Energy (keV) } & \multirow[t]{2}{*}{ Country } & \multirow[t]{2}{*}{ References } \\
\hline & 662 & 1173 & 1332 & & \\
\hline Natural bentonite & 0.079 & 0.060 & 0.057 & Egypt & Present work \\
\hline High consistency concrete & Range $(0.079-0.080)$ & Range (0.056-0.058) & Range (0.053-0.055) & Turkey & Gökçe et al. [47] \\
\hline Polyboron & 0.087 & 0.066 & 0.062 & Bangladesh & Biswas et al. [36] \\
\hline Ordinary concrete & 0.078 & 0.059 & 0.055 & & \\
\hline Pure polyethylene & 0.089 & 0.067 & 0.063 & & \\
\hline Borated polyethylene & 0.082 & 0.062 & 0.058 & & \\
\hline Water & 0.086 & 0.066 & 0.061 & & \\
\hline Beach soil & 0.076 & 0.056 & 0.052 & Bangladesh & Alam et al. [23] \\
\hline Cement & 0.071 & 0.050 & 0.047 & & \\
\hline Sand & 0.077 & 0.058 & 0.054 & & \\
\hline Bricks & 0.074 & 0.055 & 0.051 & & \\
\hline Concrete & 0.078 & 0.059 & 0.055 & & \\
\hline Gypsum & 0.058 & 0.041 & 0.039 & & \\
\hline Mosaic stone & 0.076 & 0.058 & 0.054 & & \\
\hline Limestone & 0.060 & 0.042 & 0.040 & & \\
\hline Green marble & 0.078 & 0.057 & 0.054 & India & Obaid et al. [27] \\
\hline Jet black granite & 0.077 & 0.058 & 0.055 & & \\
\hline Telephone black granite & 0.077 & 0.058 & 0.054 & & \\
\hline Cuddapah limestone & 0.078 & 0.058 & 0.055 & & \\
\hline White marble & 0.078 & 0.058 & 0.053 & & \\
\hline Pink marble & 0.078 & 0.058 & 0.055 & & \\
\hline Olivine basalt & 0.076 & 0.059 & 0.055 & & \\
\hline Barium-bismuth-borosilicate glasses & range $(0.072-0.084)$ & range $(0.053-0.057)$ & Range $(0.050-0.052)$ & Iran & Bagheri et al. [13] \\
\hline Polymer (nylon 6) & 0.086 & 0.065 & 0.061 & India & More et al. [54] \\
\hline
\end{tabular}
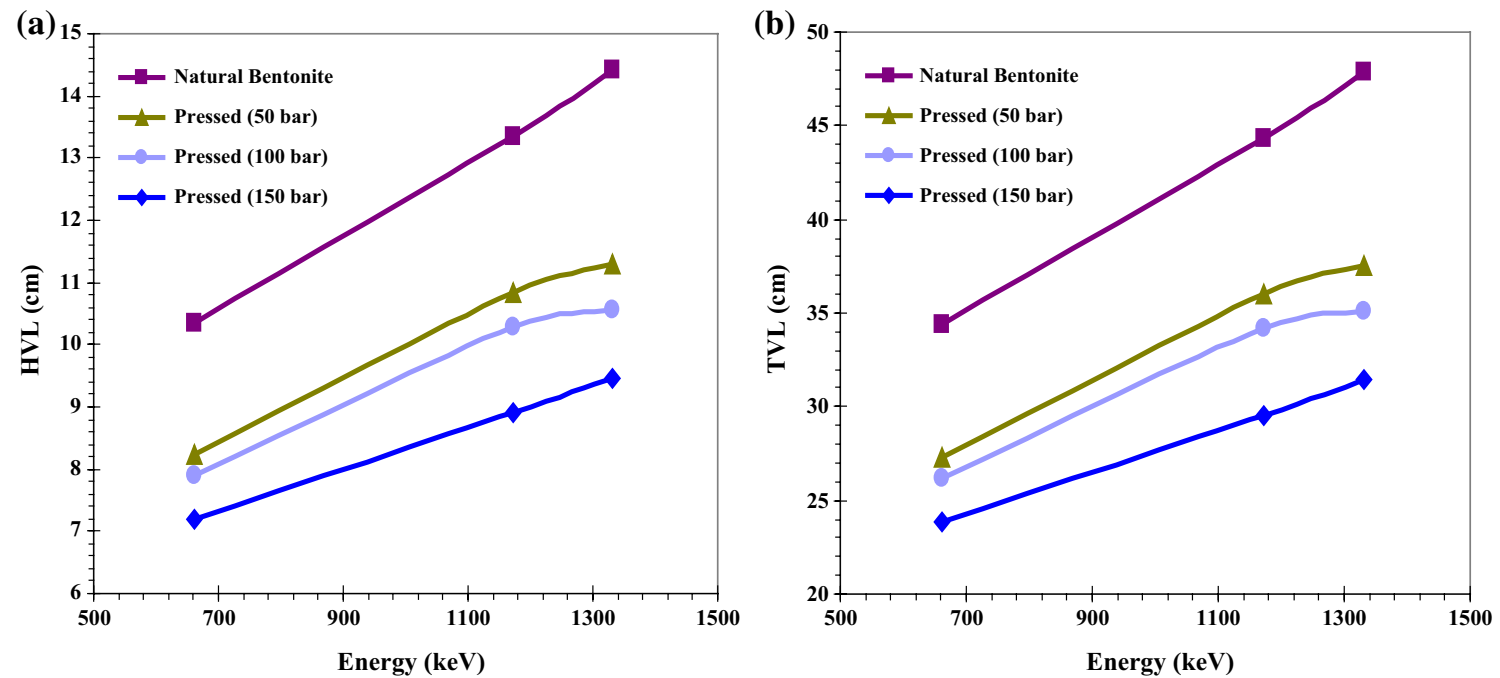

Fig. 13 Variations of a HVL and $\mathbf{b}$ TVL with energy for different bentonite clay forms 


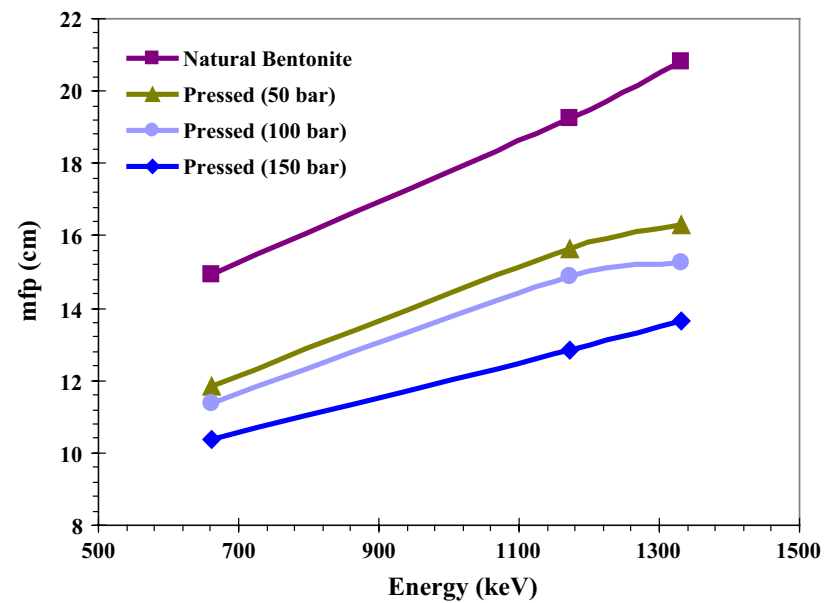

Fig. 14 Mean free path versus photon energy for different forms of bentonite clay

Open Access This article is distributed under the terms of the Creative Commons Attribution 4.0 International License (http://creativeco mmons.org/licenses/by/4.0/), which permits unrestricted use, distribution, and reproduction in any medium, provided you give appropriate credit to the original author(s) and the source, provide a link to the Creative Commons license, and indicate if changes were made.

\section{References}

1. Adams, J.H., Hathaway, D.H., Watts, R.N.: Revolutionary concepts of radiation shielding for human exploration of space. NASA/TM-2005-213688 (2005)

2. Xu, S.Q., Bourham, M., Rabiei, A.: A novel ultra-light structure for radiation shielding. Mater. Des. 4, 2140-2146 (2010)

3. Hayashi, T., Tobita, K., Nakamori, Y.J., Orimo, S.: Advanced neutron shielding material using zirconium borohydride and zirconium hydride. J. Nucl. Mater. 386, 119-121 (2009)

4. Meng, L.M., Ze, L.Y., Xing, Y.H., Ming, Z.Z.: Electrospun cerium nitrate/polymer composite fibres: synthesis, characterization and fibre-division model. Chin. Phys. B 20(4), 048101 (2009). https ://doi.org/10.1088/1674-1056/20/4/048101

5. Al-Hamarneh, I.F.: Investigation of gamma-ray shielding effectiveness of natural marble used for external wall cladding of buildings in Riyadh, Saudi Arabia. Res. Phys. 7, 1792-1798 (2017)

6. John, W.C., Joseph, G.S., Jeffrey, H.: Structural/radiation-shielding epoxies NASA Tech Briefs LAR-16874-1 (2009)

7. Xing, Y.X., Meng, L.M., He, Y., Ze, L.Y., Xin, S.: Electrical resistance response of polyaniline films to water, ethanol, and nitric acid solution Chin. Phys. B 19(8), 088105 (2010). https:// doi.org/10.1088/1674-1056/19/8/088105

8. Harish, V., Nagaiah, N., Prabhu, T.N., Varughese, K.T.: Preparation and characterization of lead monoxide filled unsaturated polyester based polymer composites for gamma radiation shielding applications. J. Appl. Polym. Sci. 112, 1503-1508 (2009)

9. Gaaz, T.S., Sulong, A.B., Akhtar, M.N., Kadhum, A.A.H., Mohamad, A.B., Al-Amiery, A.A.: Properties and applications of polyvinyl alcohol, halloysite nanotubes and their nanocomposites. Molecules 20, 22833-22847 (2015)

10. Aslam, M., Kalyar, M.A., Raza, Z.A.: Polyvinyl alcohol: a review of research status and use of polyvinyl alcohol based nanocomposites. Polym. Eng. Sci. Wiley Online Library (2018). https://doi.org/10.1002/pen.24855

11. Pour, G.B., Aval, L.F., Mirzaee, M.: Flexible graphene supercapacitor based on the PVA electrolyte and $\mathrm{BaTiO}_{3} / \mathrm{PEDOT}$ :PSS composite separator. J. Mater. Sci. Mater. Electr. 29, 17432-17437 (2018)

12. Aval, L.F., Ghoranneviss, M., Pour, G.B.: High-performance supercapacitors based on the carbon nanotubes, grapheme and graphite nanoparticles electrodes. Heliyon 4, e00862 (2018). https ://doi.org/10.1016/j.heliyon.2018.e00862

13. Bagheri, R., Moghaddam, A.K., Yousefnia, H.: Gamma ray shielding study of barium-bismuth-borosilicate glasses as transparent shielding materials using MCNP-4C Code, XCOM program, and available experimental data. Nucl. Eng. Technol. 49, 216-223 (2017)

14. Hussain, R., Haq, Z.U., Mohammad, D.: A study of the shielding properties of poly ethylene glycol-lead oxide composite. J. Islam. Acad. Sci. 10(3), 81-84 (1997)

15. Sayyed, M.I., AlZaatreh, M.Y., Dong, M.G., Zaid, M.H.M., Matori, K.A., Tekin, H.O.: A comprehensive study of the energy absorption and exposure buildup factors of different bricks for gamma-rays shielding. Res. Phys. 7, 2528-2533 (2017)

16. Oto, B., Yildiz, N., Korkut, T., Kavaz, E.: Neutron shielding qualities and gamma ray buildup factors of concretes containing limonite ore. Nucl. Eng. Des. 293, 166-175 (2015)

17. Bagheri, R., Shirmardi, S.P., Adeli, R.: Study on gamma-ray shielding characteristics of lead oxide, barite, and boron ores using MCNP-4C Monte Carlo code and experimental data. J. Test. Eval. 45(6), 2259-2266 (2017)

18. Bagheri, R., Moghaddam, A.K., Shirmardi, S.P., Azadbakht, B., Salehi, M.: Determination of gamma-ray shielding properties for silicate glasses containing $\mathrm{Bi}_{2} \mathrm{O}_{3}, \mathrm{PbO}$, and $\mathrm{BaO}$. J. Non Cryst. Solids 479, 62-71 (2018)

19. Dong, M.G., El-Mallawany, R., Sayyed, M.I., Tekin, H.O.: Shielding properties of $80 \mathrm{TeO}_{2}-5 \mathrm{TiO}_{2}-(15-\mathrm{x}) \mathrm{WO}_{3}-\mathrm{xAnOm}$ glasses using WinXCom and MCNP5 code. Radiat. Phys. Chem. 141, 172-178 (2017)

20. Awasarmol, V.V., Gaikwad, D.K., Raut, S.D., Pawar, P.P.: Photon interaction study of organic nonlinear optical materials in the energy range 122-1330 keV. Radiat. Phys. Chem. 130, 343-350 (2017)

21. Han, I., Kolaylı, H., Şahin, M.: Determination of mass attenuation coefficients for natural minerals from different places of Turkey. Int. J. Phys. Sci. 6(20), 4798-4801 (2011)

22. Al-Masri, M.S., Hasan, M., Al-Hamwi, A., Amin, Y., Doubal, A.W.: Mass attenuation coefficients of soil and sediment samples using gamma energies from 46.5 to $1332 \mathrm{keV}$. J. Environ. Radioact. 116, 28-33 (2013)

23. Alam, M.N., Miah, M.M.H., Chowdhury, M.I., Kamal, M., Ghose, S., Rahman, R.: Attenuation coefficients of soils and some building materials of Bangladesh in the energy range 276-1332 keV. Technical note. Appl. Radiat. Isot. 54(6), 973-976 (2001)

24. Akkurt, I., Altindag, R., Onargan, T., Basyigit, C., Kılıncarslan, S., Kun, M., Mavi, B., Güney, A.: The properties of various igneous rocks for $\gamma$-ray shielding. Constr. Build. Mater. 21(12), 2078$2082(2007)$

25. Karabul, Y., Susam, L.A., İçelli, O., Eyecioğlu, Ö.: Computation of EABF and EBF for basalt rock samples. Nucl. Instrum. Methods Phys. Res. Sect. A Accel. Spectrom. Detect. Assoc. Equip. 797, 29-36 (2015)

26. Obaid, S.S., Gaikwad, D.K., Pawar, P.P.: Determination of gamma ray shielding parameters of rocks and concrete. Radiat. Phys. Chem. 144, 356-360 (2018)

27. Obaid, S.S., Sayyed, M.I., Gaikwad, D.K., Pawar, P.P.: Attenuation coefficients and exposure buildup factor of some rocks for 
gamma ray shielding applications. Radiat. Phys. Chem. 148, 86-94 (2018)

28. Bagheri, R., Moghaddam, A.K., Yousefi, A.: Gamma-ray shielding study of light to heavyweight concretes using MCNP-4C code. Nucl. Sci. Technol. 28, 15 (2017)

29. Sharifi, Sh, Bagheri, R., Shirmardi, S.P.: Comparison of shielding properties for ordinary, barite, serpentine and steel-magnetite concretes using MCNP-4C code and available experimental results. Ann. Nucl. Energy 53, 529-534 (2013)

30. Mann, K.S., Singla, J., Kumar, V., Sidhu, G.S.: Investigations of mass attenuation coefficients and exposure buildup factors of some low-Z building materials. Ann. Nucl. Energy 43, 157-166 (2012)

31. Mann, K.S., Kaur, B., Sidhu, G.S., Kumar, A.: Investigations of some building materials for $\gamma$-rays shielding effectiveness. Radiat. Phys. Chem. 87, 16-25 (2013)

32. Reda, S.M.: Gamma ray shielding by a new combination of aluminum, iron, copper and lead using MCNP5. Arab. J. Nucl. Sci. Appl. 94(4), 211-217 (2016)

33. El-Fiki, S., El Kameesy, S.U., Nashar, D.E.E., Abou- Leila, M.A., El-Mansy, M.K., Ahmed, M.: Influence of bismuth contents on mechanical and gamma ray attenuation properties of silicone rubber composite. Int. J. Adv. Res. 3(6), 1035-1039 (2015)

34. Gounhalli, S.G., Shantappa, A., Hanagodimath, S.M.: Studies on mass attenuation coefficient, effective atomic numbers and electron densities of some narcotic drugs in the energy range $1 \mathrm{KeV}-$ 100GeV. IOSR J. Appl. Phys. (IOSR-JAP) 2(4), 40-48 (2012)

35. Tekin, H.O., Singh, V.P., Kara, U., Manıcı, T., Altunsoy, E.E.: Investigation of nanoparticle effect on radiation shielding property using Monte Carlo method. CBU J. Sci. 12(2), 195-199 (2016)

36. Biswas, R., Sahadath, H., Mollah, A.S., Huq, M.F.: Calculation of gamma-ray attenuation parameters for locally developed shielding material: polyboron. J. Radiat. Res. Appl. Sci. 9, 26-34 (2016)

37. Kim, J., Seo, D., Lee, B.C., Seo, Y.S., Miller, W.H.: Nano-W dispersed gamma radiation shielding materials. Adv. Eng. Mater. (2014). https://doi.org/10.1002/adem.201400127

38. Selvi, S.S.T., Linet, J.M., Sagadevan, S.: Influence of CTAB surfactant on structural and optical properties of $\mathrm{CuS}$ and $\mathrm{CdS}$ nanoparticles by hydrothermal route. J. Exp. Nanosci. 13(1), 130-143 (2018)

39. Barhoum, A., Assche, G.V., Maklouf, A.S.H., Terryn, H., Baert, K., Delplancke, M.-P., Sheikh, S.M., Rahier, H.: A green, simple chemical route for the synthesis of pure nanocalcite crystals. Cryst. Growth Des. (2014). https://doi.org/10.1021/cg501121t

40. Tang, Q., Chen, X., Hu, W., Zhou, G.: Microwave-assisted hydrothermal synthesis of hexagonal columnar-shaped calcium carbonate. Adv. Mater. Res. 239-242, 1643-1648 (2011)

41. Mohseni, K.: Characterization of precipitated calcium carbonate (PCC) compounds on the basis of powder X-ray diffraction data. Ph.D., Universität Karlsruhe (TH), Iran-Tehran (2007)

42. Boussaa, S.A., Kheloufi, A., Zaourar, N.B., Kerkar, F.: Valorization of Algerian sand for photovoltaic application. Acta Phys. Pol., A 130(1), 133-137 (2016)

43. Suriyanarayanan, N., Nithin, K.V.K., Bernardo, E.: Mullite glass ceramics production from coal ash and alumina by high temperature plasma. Journal of Non-Oxide Glasses 1(4), 247-260 (2009)

44. Khorsand, A.Z., Abd Majid, W.H., Abrishami, M.E., Ramin, Y.: $\mathrm{X}$-ray analysis of $\mathrm{ZnO}$ nano-particles by Williamson-Hall and size-strain polt methods. Solid State Sci. 13, 251-256 (2011)

45. Abdi, F., Derayat, J., Esmaeili, R., Fattahi, N., Sharafi, H., Abdi, A.: X-ray transmission through nanostructured and microstructured tin oxide materials. Int. J. Pharm. Technol. 8(3), 1892518933 (2016)

46. Luisa, F., Duncan, S.: Introduction to Nanoscience and Nanotechnologyies, Module 1, Fundamental Concepts in Nanoscience and Nanotechnologies, Interdisciplinary Nanoscience Center (INANO). Aarhus University, Denmark (2010)

47. Gökçe, H.S., Öztürk, B.C., Çam, N.F., Andiç-Çakır, Ö.: Gammaray attenuation coefficients and transmission thickness of high consistency heavyweight concrete containing mineral admixture. Cem. Concr. Compos. 92, 56-69 (2018)

48. Baker, M.I., Walsh, S.P., Schwartz, Z., Boyan, B.D.: A review of polyvinyl alcohol and its uses in cartilage and orthopedic applications. J. Biomed. Mater. Res., Part B (2012). https://doi. org/10.1002/jbm.b.32694

49. Malekie, S., Hajiloo, N.: Comparative study of micro and nano size $\mathrm{WO}_{3} / \mathrm{E} 44$ epoxy composite as gamma radiation shielding using MCNP and experiment. Chin. Phys. Lett. 34(10), 108102 (2017)

50. El-Sawy, A.A.: Performance of nanoparticle materials on radiation shielding properties using Mont Carlo method. Int. J. Eng. Sci. (IJES) 6(6), 74-82 (2017)

51. Berger, M.J., Hubbell, J.H., XCOM: Photon Cross Sections Data Base, Web version, 1.2. National Institute of Standards and Technology, Gaithersburg (1999) (Originally published as NBSIR 87-3597 "XCOM: Photon cross sections on a personal computer"). http://physics.nist.gov/PhysRefData/Xcom/Text/XCOM. html

52. Erdem, M., Baykara, O., Doğru, M., Kuluöztürk, F.: A novel shielding material prepared from solid waste containing lead for gamma ray. Radiat. Phys. Chem. 79, 917-922 (2010)

53. Berger, M.J., Hubbell, J.H., Seltzer, S.M., Chang, J., Coursey, J.S., Sukumar, R., Zucker, D.S., Olsen, K.: XCOM: Photon Cross-Section Database. (2010). http://www.nist.gov/pml/data/xcom/index .cfm.us

54. More, C.V., Bhosale, R.R., Pawar, P.P.: Detection of new polymer materials as gamma-ray-shielding materials. Radiat. Eff. Defects Solids (2017). https://doi.org/10.1080/10420150.2017.1336765

55. Dogra, M., Singh, K.J., Kaur, K., Anand, V., Kaur, P.: Gamma ray shielding and structural properties of $\mathrm{Bi}_{2} \mathrm{O}_{3}-\mathrm{B}_{2} \mathrm{O}_{3}-\mathrm{Na}_{2} \mathrm{WO}_{4}$ glass system. Univ. J. Phys. Appl. 11(5), 190-195 (2017)

Publisher's Note Springer Nature remains neutral with regard to jurisdictional claims in published maps and institutional affiliations. 